\title{
FATORES INFLUENTES NO ESTILO DE VIDA E NA SAÚDE DOS IDOSOS BRASILEIROS: UMA REVISÃO INTEGRATIVA
}

\author{
Rubenyta Martins Podmelle ${ }^{1}$ \\ Rogério Dubosselard Zimmermann²
}

resumo

O envelhecimento da população é uma realidade e diversos são os fatores que influenciam na saúde dos indivíduos que estão envelhecendo, como o estilo de vida, sendo um dos pontos mais importantes para se apresentar uma boa saúde. Verificado o envelhecimento populacional no Brasil e a grande influência do estilo de vida na saúde do idoso, foi realizada uma Revisão Integrativa no intuito de identificar quais os fatores que influenciam o estilo de vida dos idosos brasileiros e quais as consequências para sua saúde de acordo com a produção científica nacional. Foram selecionados 39 trabalhos

1 Graduada em Educação Física pela Universidade de Pernambuco e Fisioterapia pelo Centro Universitário São Miguel. Mestre em Gerontologia pela Universidade Federal de Pernambuco. Professora do Centro Universitário Brasileiro (UNIBRA-PE) e da Faculdade São Miguel (PE). E-mail: rmartins63@hotmail.com.

2 Graduado em Odontologia pela Universidade Federal de Pernambuco. Doutor em Odontologia Legal e Deontologia pela Universidade Estadual de Campinas. Professor associado do Departamento de Medicina Social e do Programa de Pós-Graduação em Gerontologia da Universidade Federal de Pernambuco (UFPE). E-mail: rdzlegal@gmail.com. 
publicados entre 2006 e 2016, nos idiomas inglês e português, advindos do portal de dados Biblioteca Virtual em Saúde (BVS) e da base de dados Scientific Electronic Library Online (SciELO), a partir da combinação dos descritores "envelhecimento", "idoso" e "estilo de vida". Verificou-se que inatividade física, tabagismo, presença de DCNT, alimentação inadequada, dislipidemia, consumo excessivo de álcool, obesidade, hipercolesterolemia, alterações na glicemia, resistência à insulina e o avanço da idade foram os fatores de risco relacionados ao estilo de vida que mais influenciaram negativamente a saúde do idoso. A autoavaliação negativa da saúde foi prevalente entre idosos fumantes, sedentários, com três ou mais DCNT, que fazem uso de cinco medicamentos ou mais e que não consumiam frutas e verduras. Conclui-se, então, que os idosos brasileiros apresentam estilo de vida inadequado devido a diferentes fatores, culminando em baixas condições de saúde e autoavaliações negativas da saúde, o que necessita urgentemente de atenção.

palavras-chave

Saúde do Idoso. Estilo de Vida. Fatores de Risco.

\section{Introdução}

O envelhecimento populacional é um fenômeno global que ocorre em ritmo acelerado, acontecendo de maneira mais intensa nos países em desenvolvimento (FAZZIO, 2012). No Brasil, os idosos constituem o segmento etário que mais aumenta, passando de 14,2 milhões, no ano 2000, para 41,5 milhões em 2030 (IBGE, 2014). Diversos são os fatores que influenciam na saúde dos indivíduos que estão envelhecendo, existem os fatores intrínsecos, como sexo, idade e genética, e, principalmente, os fatores extrínsecos, que se associam ao estilo de vida (SOUZA et al., 2016) que, de acordo com a Organização Mundial da Saúde (OMS, 2006), é um dos componentes mais importantes para se apresentar uma boa saúde. Petroski e Pelegrini (2009) conceituam o estilo de vida como um modelo comportamental que pode oferecer grande efeito na saúde dos indivíduos, ou seja, as decisões tomadas afetarão as condições de saúde de alguma maneira, tornando o estilo de vida determinante para a qualidade do envelhecimento.

Na medida em que a idade média da população aumenta, mais pessoas sofrerão de algum tipo de doença (WHO, 2013), tais como Doenças Crônicas Não Transmissíveis (DCNT), as quais, segundo Focchesatto, Rockett e Perry (2015), são responsáveis por significativo número de mortes entre idosos. Prevenção e tratamento de DCNT e incapacidades funcionais relacionadas 
ao envelhecimento dependem justamente, em grande parte, do estilo de vida que o indivíduo apresenta (JOBIM, F.; JOBIM, E., 2015). Compreendendo a relevância das consequências de um estilo de vida insalutífero entre idosos e tendo em vista o elevado crescimento dessa população no Brasil, o presente estudo se propõe a identificar quais os fatores que influenciam o estilo de vida dos idosos brasileiros e quais as consequências para sua saúde de acordo com o conhecimento científico produzido no país.

\section{Métodos}

Trata-se de uma Revisão Integrativa, que se caracteriza como método que propicia a reunião de conhecimentos e sua inserção na prática, uma vez que agrupa diversas pesquisas em um único estudo e possibilita ao leitor a redução de alguns obstáculos para sua utilização, tornando os resultados científicos mais acessíveis (SOUZA; SILVA; CARVALHO, 2010). Tem-se como base a pergunta norteadora "Qual o estilo de vida da população idosa brasileira e como este reflete na sua saúde?".

No intuito de reduzir vieses, o portal de dados Biblioteca Virtual em Saúde (BVS) foi preferido como local de busca, uma vez que apresenta imponente alcance de bases de dados. Ainda, como estratégia de enriquecimento da coleta, o site Scientific Electronic Library Online (SciELO) foi considerado. Os critérios estabelecidos para a inclusão dos estudos foram: textos completos disponíveis, realizados no Brasil, publicados nos idiomas inglês e português entre os anos 2006 e 2016, cuja abordagem direcionou-se aos fatores que influenciam o estilo de vida e à influência do estilo de vida na saúde dos idosos brasileiros. O intervalo de dez anos foi preferido para permitir maior atualização das publicações encontradas. O levantamento dos dados ocorreu em junho de 2016, em ambos os locais de busca. Para atender a esses objetivos, foram selecionados dos descritores "envelhecimento", "idoso" e "estilo de vida".

No cruzamento dos descritores, foram disponibilizados 23.817 arquivos. Quando aplicados os critérios de inclusão, atingiu-se o quantitativo de 73 estudos, tendo 15 se repetido entre a base e o portal de dados e quatro entre as chaves de busca ("envelhecimento" AND "estilo de vida" e "idoso" AND "estilo de vida"), totalizando 54 estudos selecionados para leitura na íntegra. Após a leitura destes trabalhos, 39 foram eleitos para compor a amostra desta revisão a partir dos seguintes critérios de exclusão: artigos de revisão, validação de instrumentos, trabalhos sobre condições de vida na infância que refletem na velhice e amostras representadas por outros grupos populacionais (opinião de profissionais de saúde, por exemplo). 
O corpus de análise do presente trabalho é composto apenas por estudos brasileiros, no entanto, nem todos foram publicados em português (87,17\%). Dentre os 39 estudos incluídos, apenas um é em formato de dissertação, mas compõe-se por dois artigos. O ano de publicação mais frequente foi 2015 (17,94\%), seguido por 2014 e 2010 (15,38\% cada).

Quanto aos periódicos de publicação, foi detectado um total de 23 periódicos, tendo o Caderno de Saúde Pública maior número de artigos no presente referencial (20,5\%). Em relação à distribuição dos trabalhos de acordo com o eixo temático do periódico, a área de saúde pública $(41,02 \%)$ merece destaque. A maioria das publicações foi proveniente do estado de São Paulo (23,07\%), como mostra na Tabela 1.

Tabela 1 - Distribuição dos estudos segundo região de origem da amostra, 2016.

\begin{tabular}{lcc}
\hline Local de origem & $\mathrm{N}^{\circ}$ & $\%$ \\
\hline Brasil (amostra representativa) & 2 & 5,12 \\
Brasilia & 2 & 5,12 \\
Goiás & 4 & 10,25 \\
Maranhão & 1 & 2,56 \\
Minas Gerais & 7 & 17,94 \\
Paraíba & 1 & 2,56 \\
Paraná & 1 & 2,56 \\
Pernambuco & 1 & 2,56 \\
Piauí & 1 & 2,56 \\
Rio de Janeiro & 1 & 2,56 \\
Rio Grande do Norte & 1 & 2,56 \\
Rio Grande do Sul & 5 & 12,82 \\
São Paulo & 9 & 23,07 \\
Santa Catarina & 3 & 7,69 \\
Total & 39 & 100 \\
\hline
\end{tabular}

Fonte: Tabela elaborada pelos autores.

Os estudos também foram classificados de acordo com seu conceito metodológico: 35 estudos são quantitativos e apenas quatro qualitativos. A leitura dos trabalhos possibilitou a identificação de quatro categorias temáticas: estilo de vida e autoavaliação da saúde, fatores de risco para DCNT, atividade física e alimentação. O Quadro 1 apresenta a síntese dos trabalhos selecionados. 


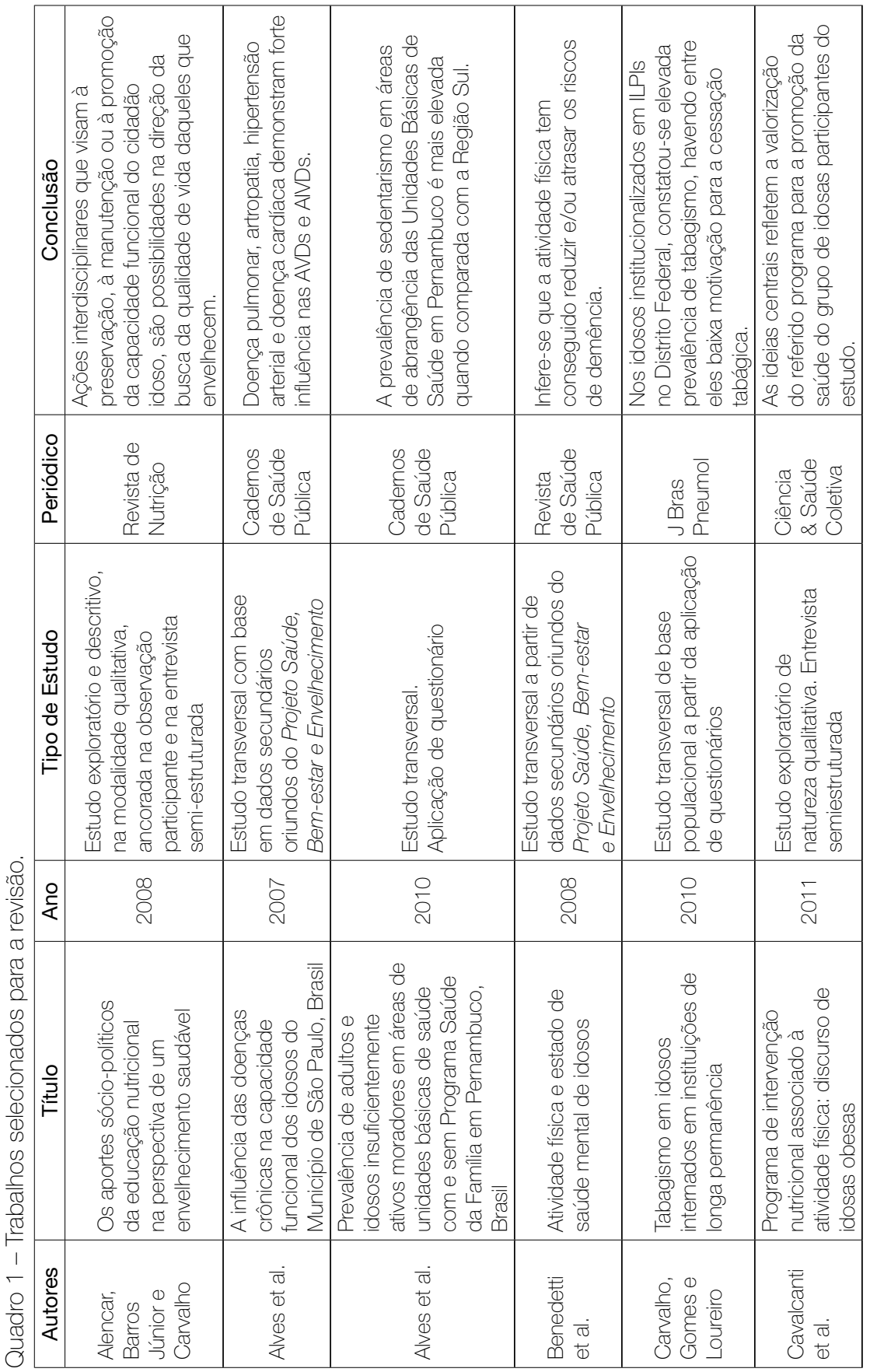


$\infty$
0
0
$\square$
$\square$
$\square$
$\square$

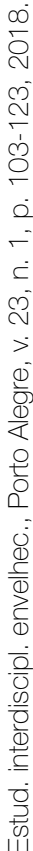

\begin{tabular}{|c|c|c|c|c|c|c|}
\hline & 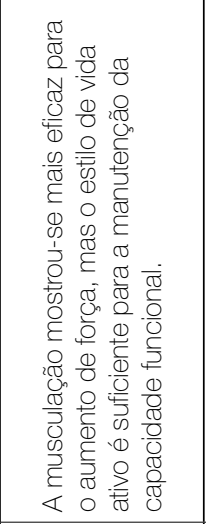 & 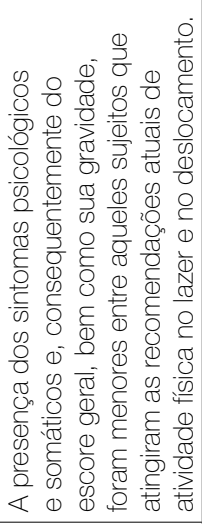 & 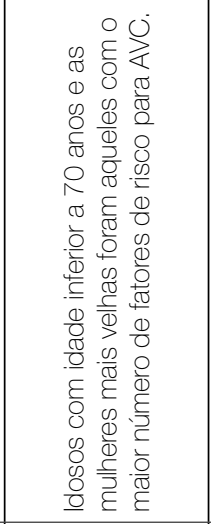 & 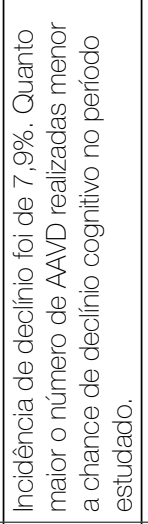 & 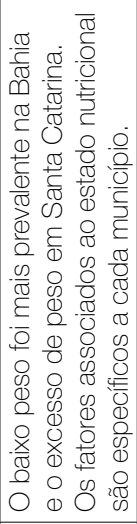 & 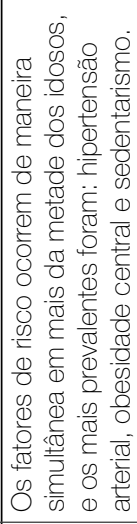 \\
\hline & 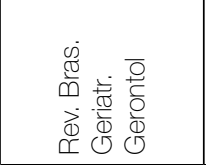 & 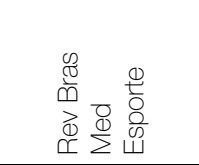 & 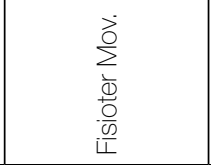 & 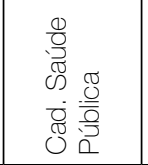 & 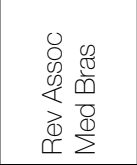 & 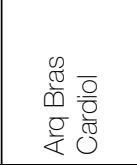 \\
\hline 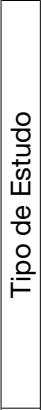 & 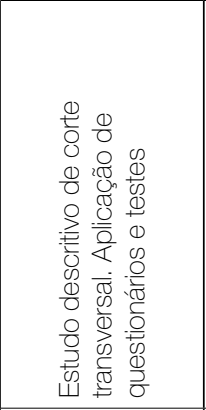 & 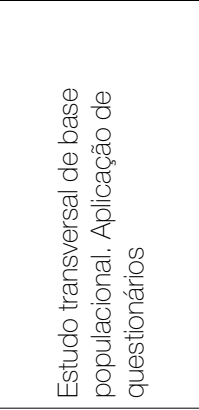 & 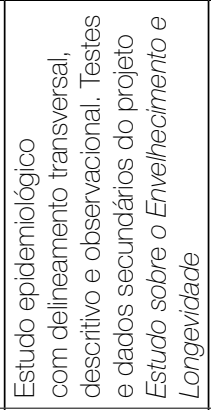 & 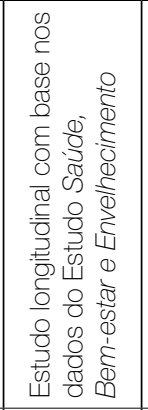 & 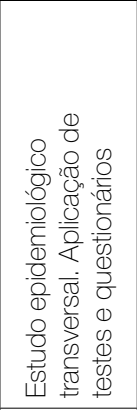 & 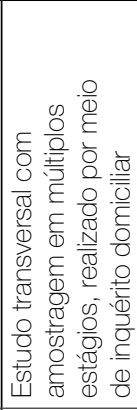 \\
\hline$\frac{2}{\frac{2}{4}}$ & $\overline{\mathrm{i}}$ & $\bar{\delta}$ & $\frac{+}{\grave{~}}$ & 5 & 5 & $\bar{\sim}$ \\
\hline 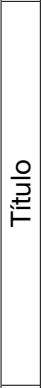 & 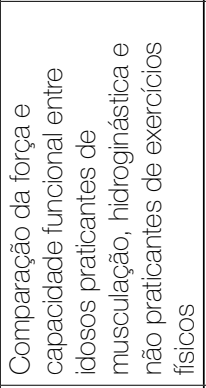 & 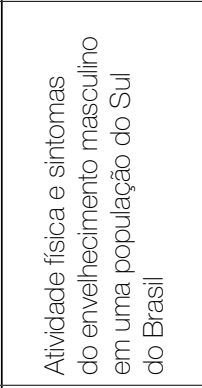 & 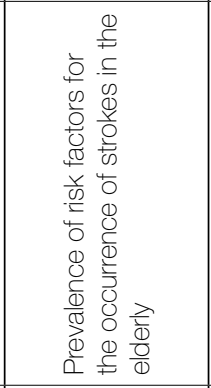 & 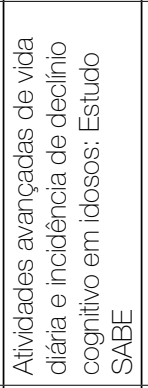 & 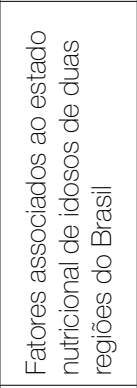 & 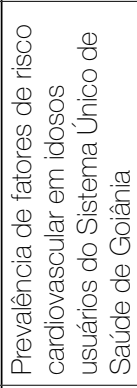 \\
\hline 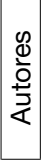 & $\begin{array}{l}\overline{0} \\
\pm \\
0 \\
0 \\
\frac{1}{0} \\
0 \\
0\end{array}$ & 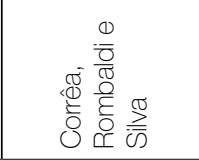 & 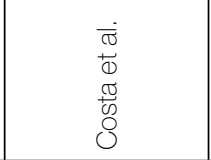 & $\begin{array}{l}\mathbb{D} \\
0 \\
\widetilde{D}\end{array}$ & $\begin{array}{l}\bar{\sigma} \\
+\mathbb{0} \\
0 \\
0 \\
D\end{array}$ & 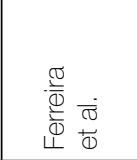 \\
\hline
\end{tabular}




\begin{tabular}{|c|c|c|c|c|c|c|}
\hline & 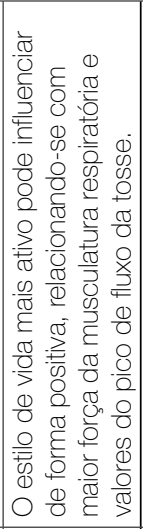 & 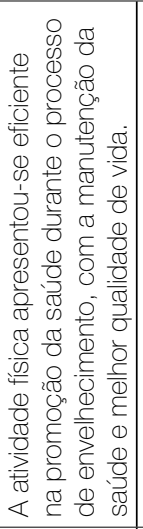 & 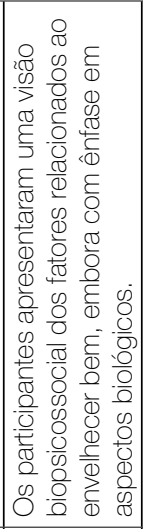 & 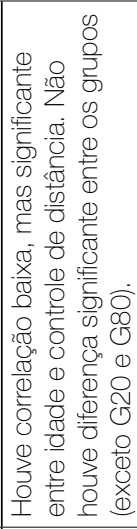 & 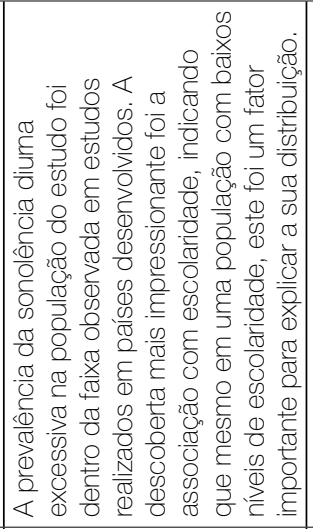 & 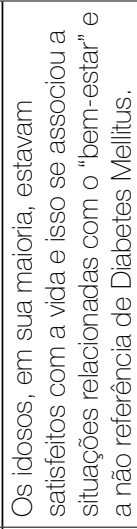 \\
\hline $\begin{array}{l}0 \\
\frac{0}{0} \\
: \frac{0}{d 0} \\
0\end{array}$ & 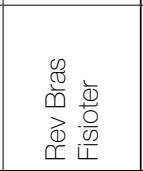 & 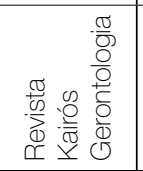 & 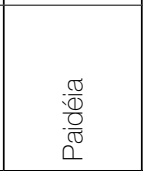 & 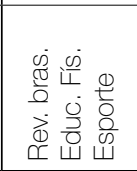 & 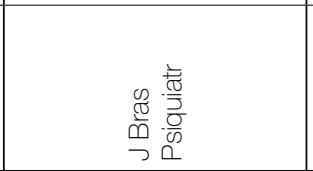 & 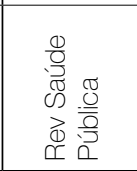 \\
\hline 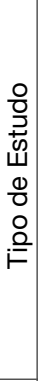 & 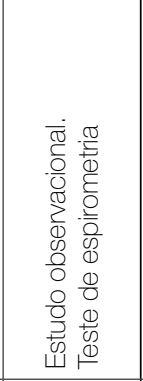 & 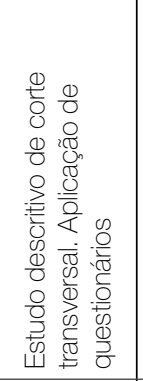 & 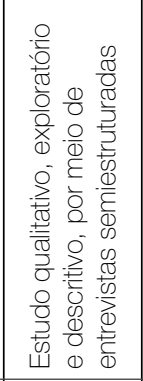 & 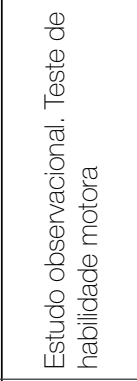 & 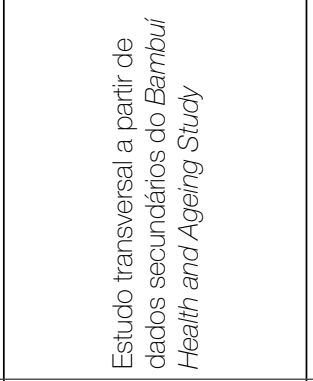 & 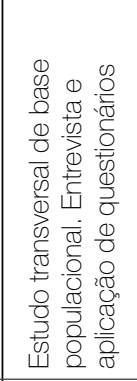 \\
\hline$\frac{O}{\frac{1}{<}}$ & $\stackrel{\circ}{\stackrel{\sim}{\alpha}}$ & $\stackrel{\nabla}{\check{\sim}}$ & $\bar{\delta}$ & 8 & 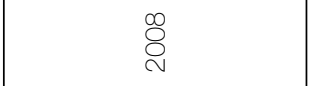 & 8 \\
\hline$\stackrel{\text { 을 }}{\stackrel{2}{*}}$ & 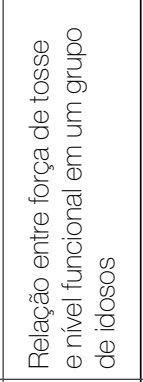 & 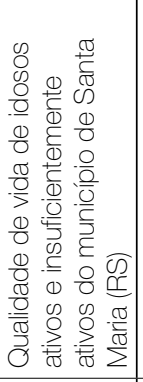 & 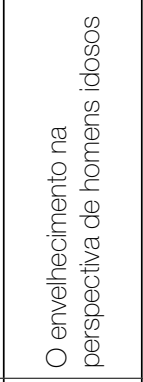 & 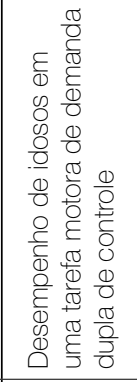 & 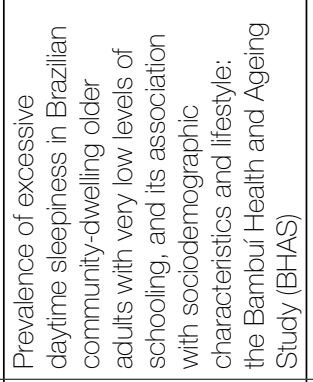 & 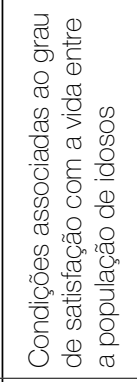 \\
\hline 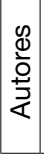 & 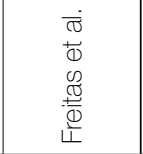 & 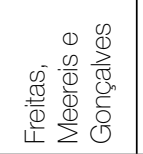 & 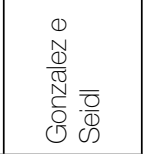 & 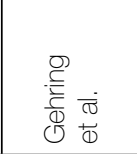 & 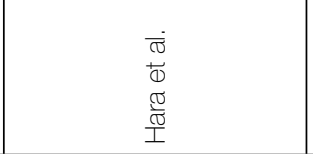 & 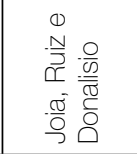 \\
\hline
\end{tabular}




\begin{tabular}{|c|c|c|c|c|c|}
\hline 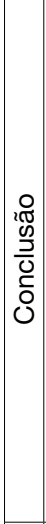 & 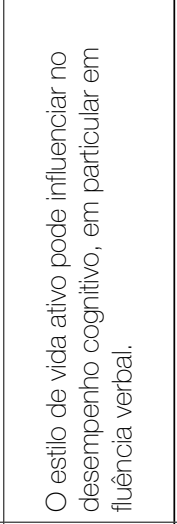 & 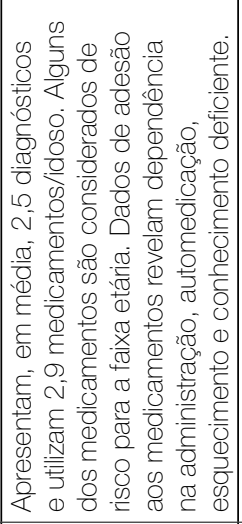 & 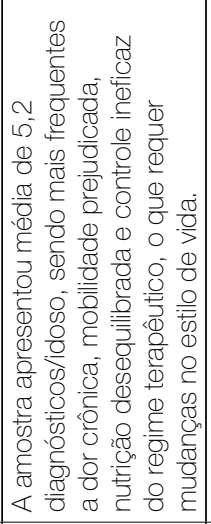 & 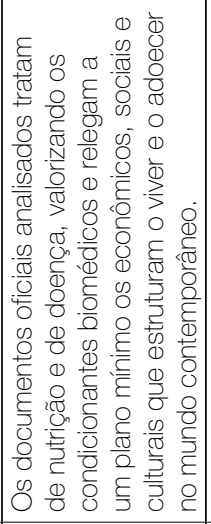 & 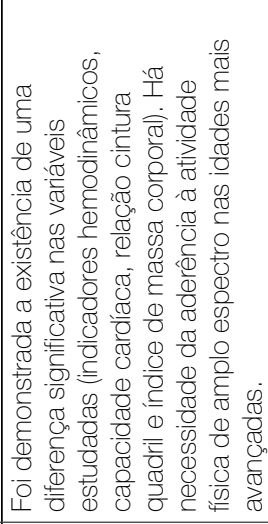 \\
\hline $\begin{array}{l}0 \\
\frac{0}{0} \\
: \frac{0}{0} \\
0 \\
0 .\end{array}$ & 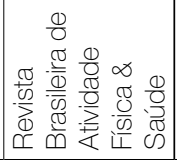 & 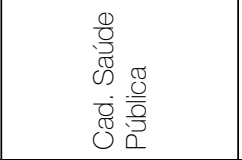 & 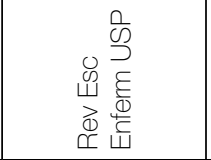 & 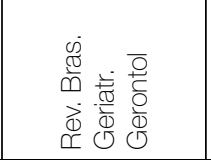 & 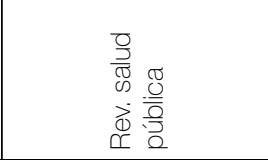 \\
\hline 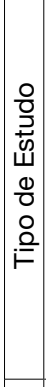 & 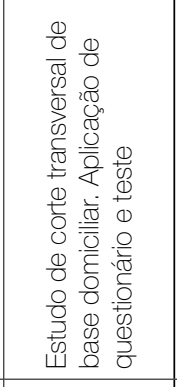 & 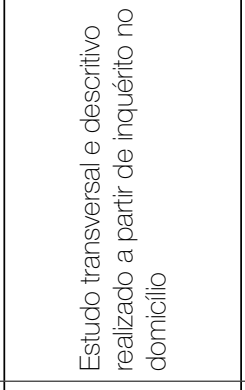 & 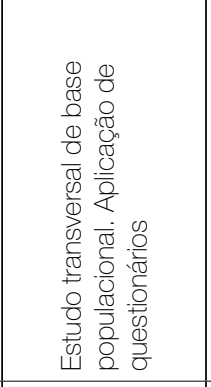 & 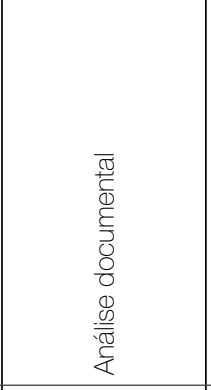 & 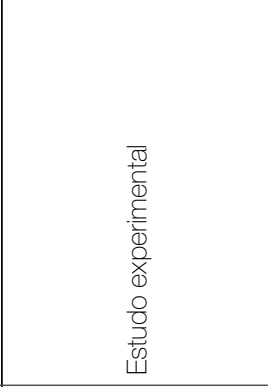 \\
\hline$\stackrel{\circ}{\frac{C}{<}}$ & $\begin{array}{l}\circ \\
\delta\end{array}$ & $\stackrel{\infty}{8}$ & $\stackrel{\circ}{\circ}$ & $\stackrel{\llcorner 0}{\delta}$ & $\stackrel{8}{8}$ \\
\hline 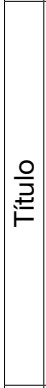 & 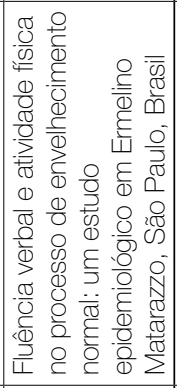 & 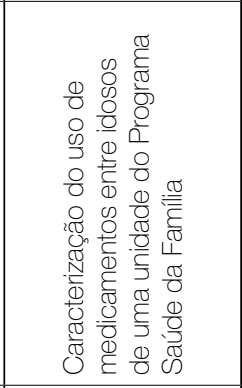 & 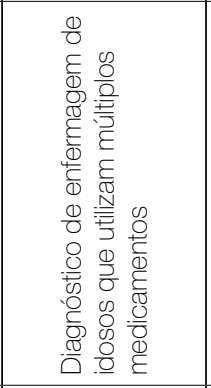 & 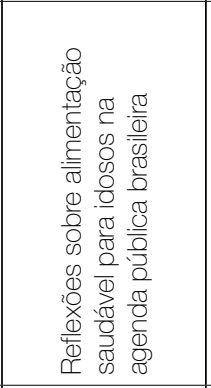 & 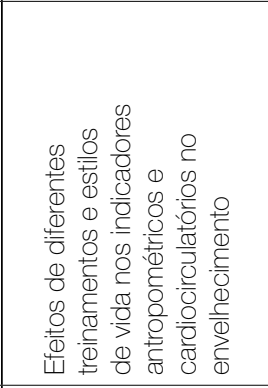 \\
\hline 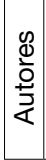 & 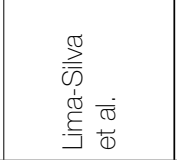 & 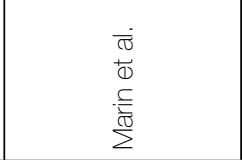 & 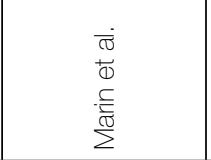 & 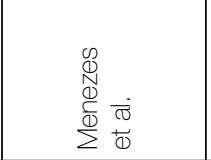 & 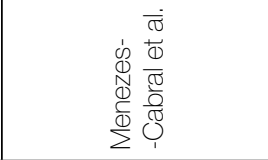 \\
\hline
\end{tabular}




\begin{tabular}{|c|c|c|c|c|c|}
\hline 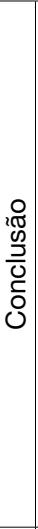 & 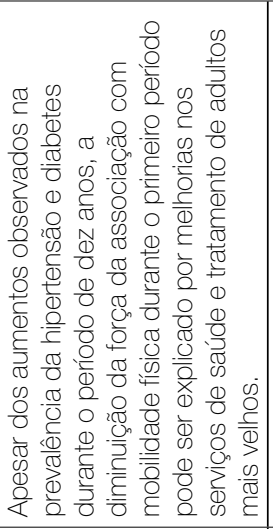 & 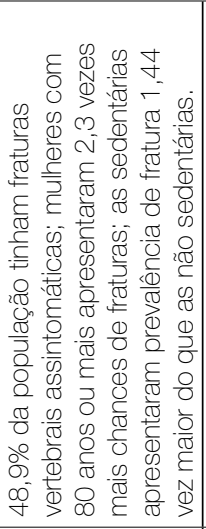 & 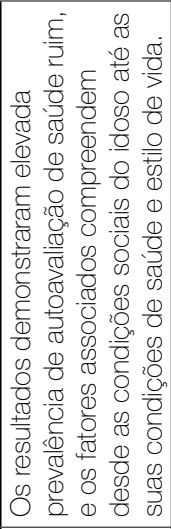 & 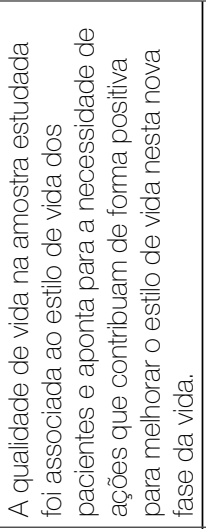 & 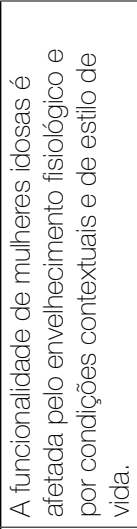 \\
\hline $\begin{array}{l}\frac{0}{0} \\
: \frac{0}{10} \\
0\end{array}$ & 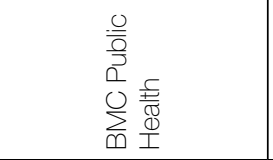 & 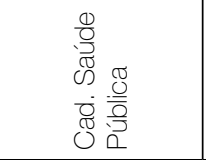 & 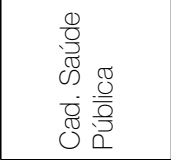 & 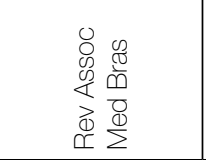 & 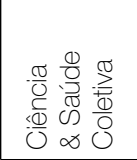 \\
\hline 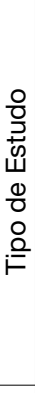 & 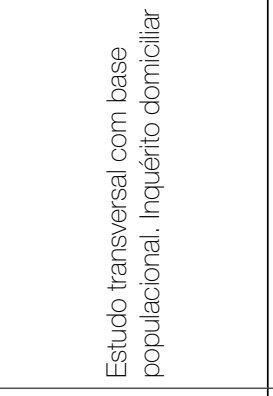 & 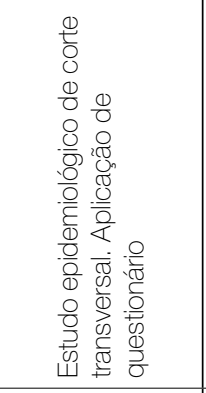 & 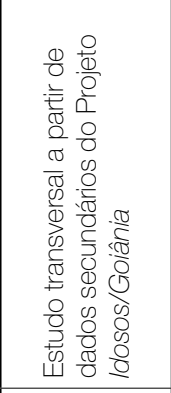 & 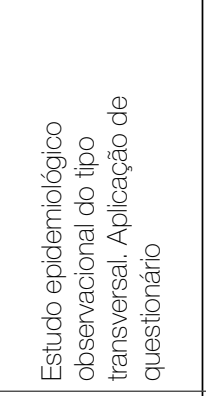 & 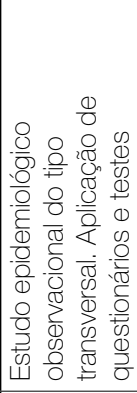 \\
\hline$\frac{\text { 원 }}{\frac{c}{4}}$ & $\stackrel{\llcorner}{\delta}$ & $\stackrel{\circ}{\circ}$ & $\bar{\delta}$ & $\begin{array}{l}\infty \\
\stackrel{8}{8} \\
\sim\end{array}$ & $\stackrel{\sim}{\tilde{\sim}}$ \\
\hline$\stackrel{\text { 을 }}{\stackrel{ \pm}{F}}$ & 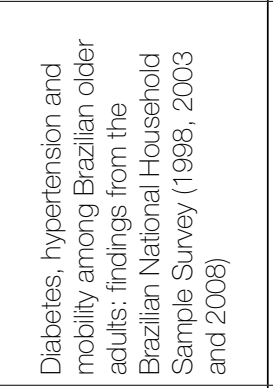 & 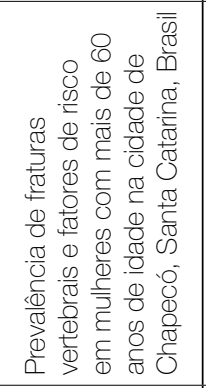 & 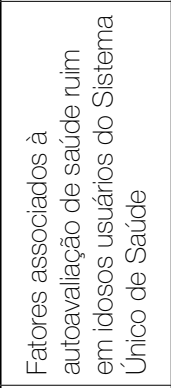 & 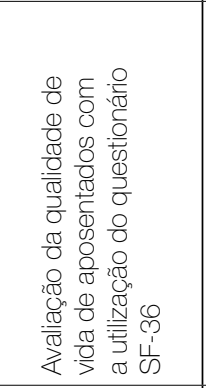 & 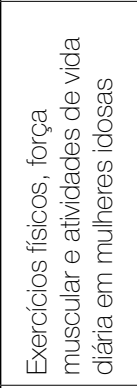 \\
\hline 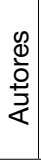 & 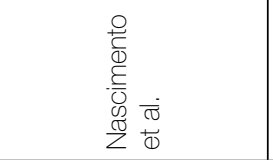 & 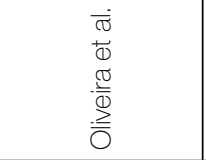 & 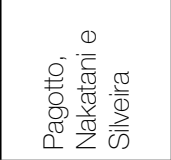 & 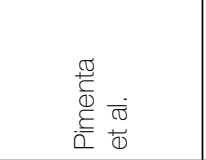 & 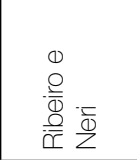 \\
\hline
\end{tabular}


0
0
0
$\square$
$\square$
$\square$

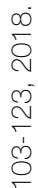

อ

ᄃ

ल

$>$

(1)

安

윰

ए

竞

음

$\frac{0}{0}$

I⿳亠口冋.

항

\begin{tabular}{|c|c|c|c|c|}
\hline 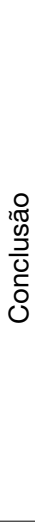 & 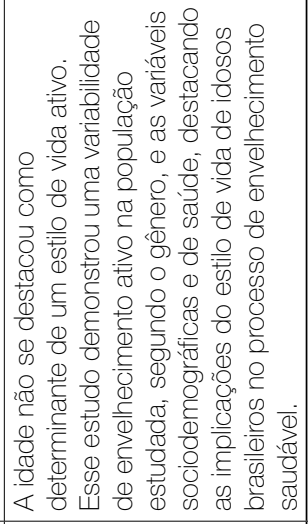 & 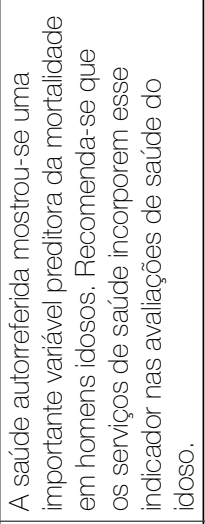 & 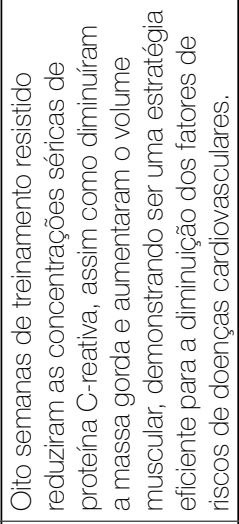 & 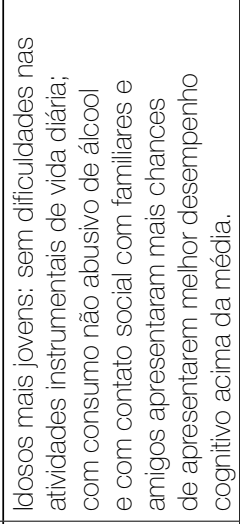 \\
\hline $\begin{array}{l}\text { O } \\
\frac{0}{0} \\
: \frac{0}{0} \\
0\end{array}$ & 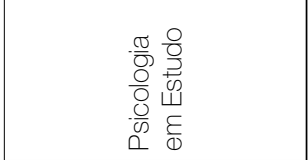 & $\begin{array}{l}\stackrel{5}{\tilde{W}} \\
\stackrel{W}{W} \\
\frac{\mathscr{D}}{0}\end{array}$ & 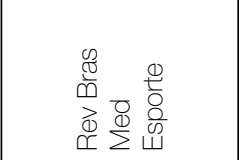 & 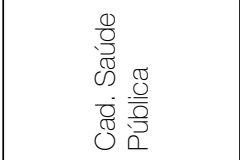 \\
\hline 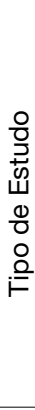 & 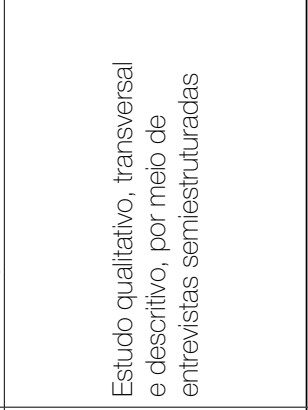 & 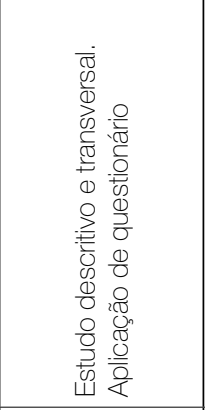 & 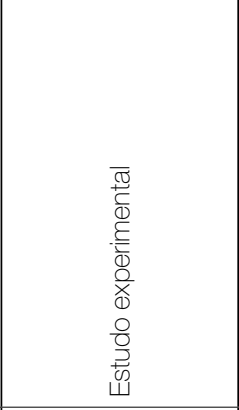 & 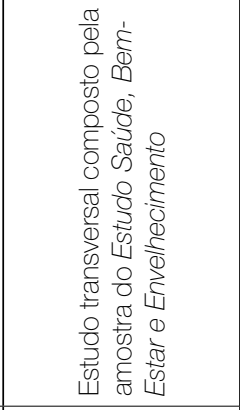 \\
\hline$\frac{O}{<}$ & ஓి & ஓి & $\frac{\llcorner 0}{\delta}$ & $\underset{\sigma}{\nabla}$ \\
\hline 을 & 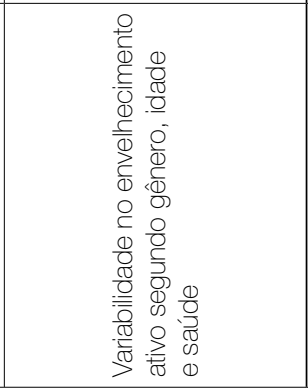 & 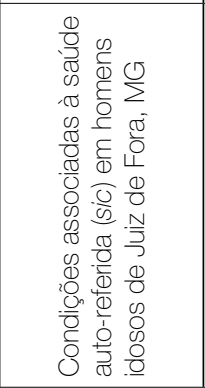 & 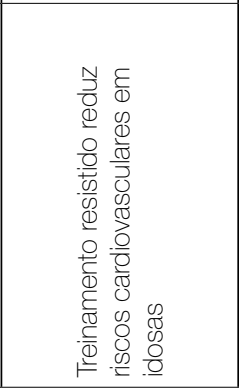 & 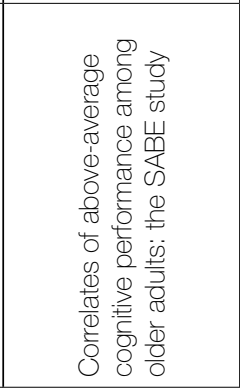 \\
\hline $\begin{array}{l}\frac{1}{0} \\
\frac{0}{2} \\
\frac{1}{2}\end{array}$ & $\begin{array}{l}\dot{\vec{\sigma}} \\
\frac{\mathbb{d}}{0} \\
\frac{0}{\overline{0}} \\
\frac{0}{\bar{\alpha}}\end{array}$ & 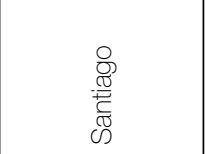 & 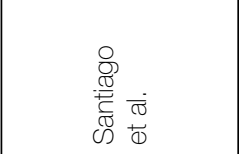 & $\begin{array}{l}\bar{\sigma} \\
\stackrel{0}{0} \\
\stackrel{\sigma}{0} \\
\stackrel{\bar{\omega}}{2}\end{array}$ \\
\hline
\end{tabular}




\begin{tabular}{|c|c|c|c|c|}
\hline 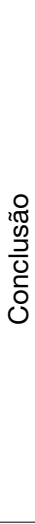 & 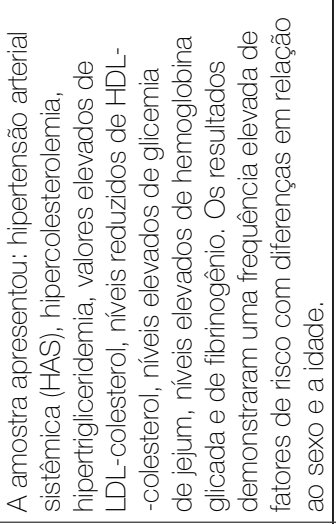 & 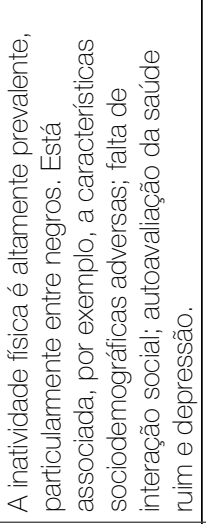 & 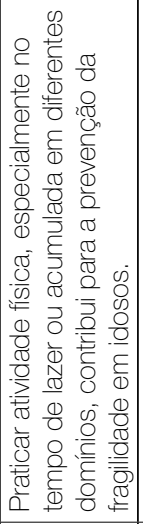 & 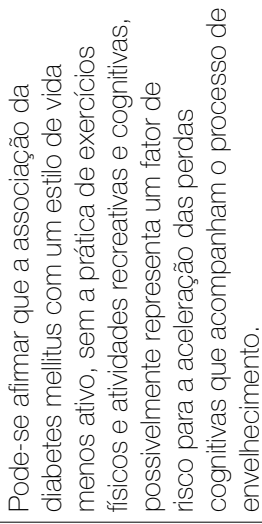 \\
\hline $\begin{array}{l}\frac{0}{0} \\
\frac{0}{0} \\
\frac{0}{0} \\
0\end{array}$ & 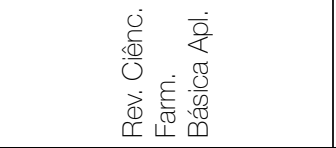 & $\begin{array}{l}山 \\
0 \\
0 \\
0 \\
0 \\
0\end{array}$ & 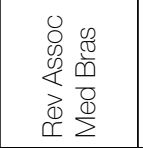 & 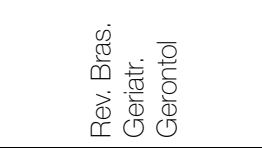 \\
\hline 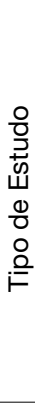 & 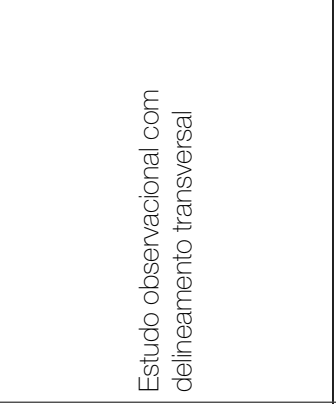 & 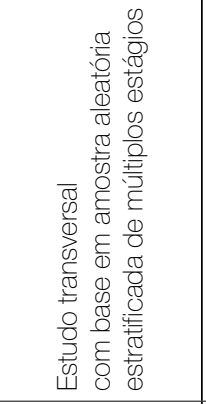 & 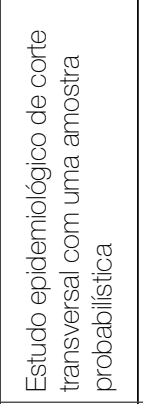 & 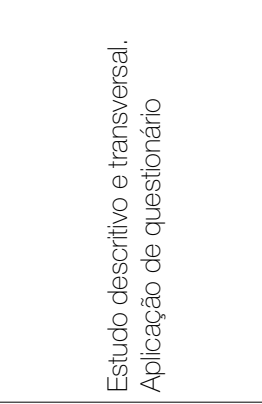 \\
\hline$\frac{\circ}{\frac{c}{4}}$ & $\stackrel{\curvearrowright}{8}$ & $\stackrel{\llcorner}{\delta}$ & $\stackrel{\sim}{\bar{\sigma}}$ & $\stackrel{\nabla}{\dot{\sim}}$ \\
\hline $\begin{array}{l}\text { 을 } \\
\text { 로 }\end{array}$ & 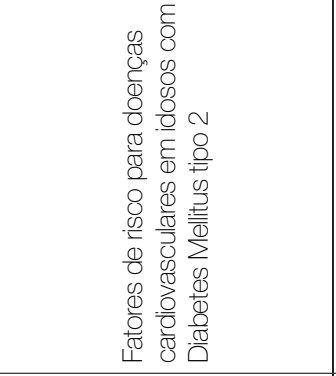 & 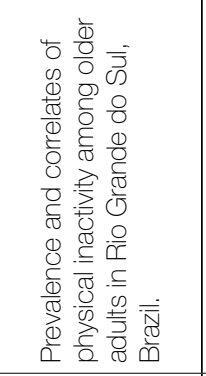 & 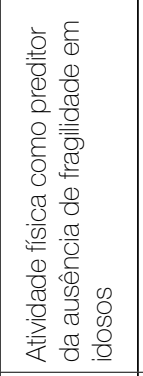 & 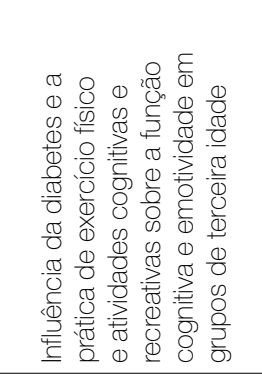 \\
\hline $\begin{array}{l}\text { क } \\
\frac{0}{0} \\
\frac{1}{3} \\
\frac{1}{4}\end{array}$ & 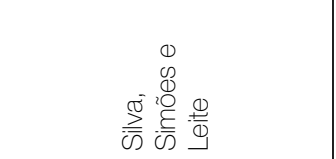 & 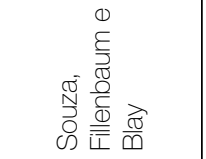 & 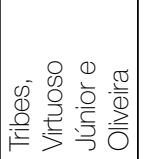 & 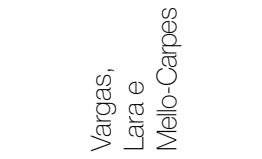 \\
\hline
\end{tabular}


0
0
0
$\square$
$\square$
$\square$

\begin{tabular}{|c|c|c|c|}
\hline 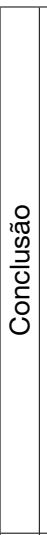 & 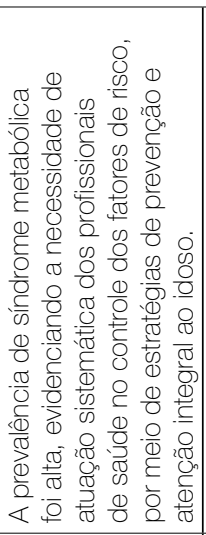 & 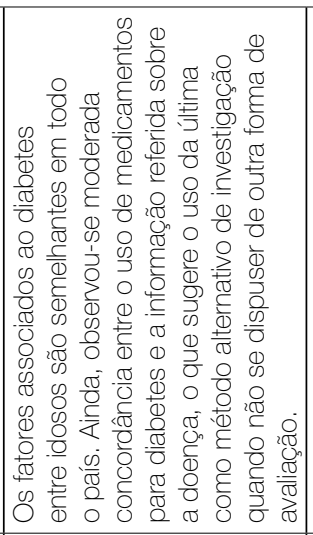 & 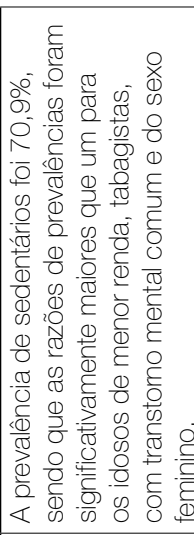 \\
\hline $\begin{array}{l}\text { O } \\
: \frac{0}{0} \\
: \frac{0}{\overline{0}} \\
0 \\
0\end{array}$ & 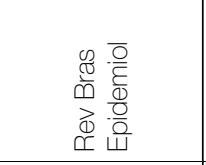 & 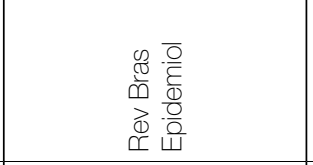 & 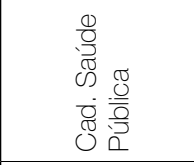 \\
\hline 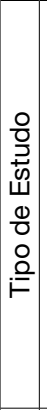 & 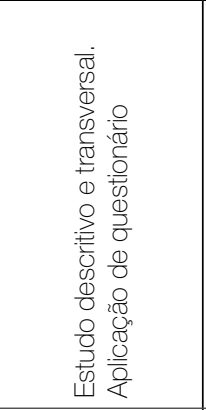 & 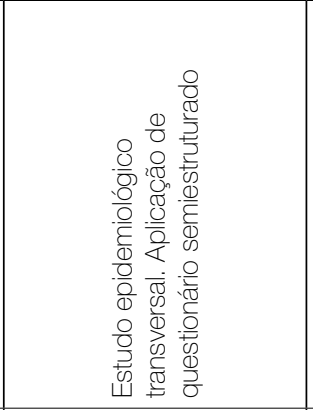 & 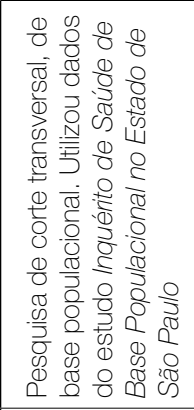 \\
\hline$\stackrel{?}{\frac{9}{4}}$ & $\stackrel{\nabla}{\dot{\sigma}}$ & $\stackrel{\llcorner}{\Sigma}$ & ஓे \\
\hline$\stackrel{0}{\stackrel{ }{3}}$ & 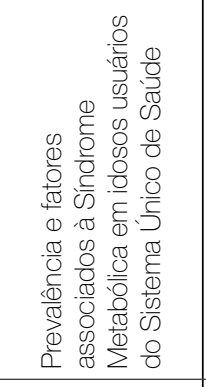 & 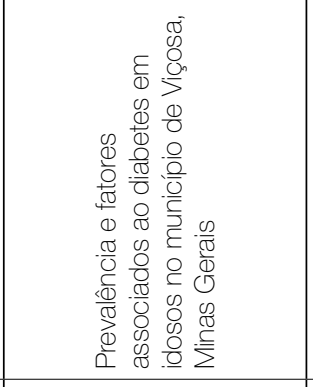 & 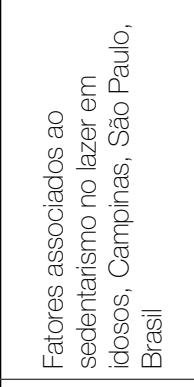 \\
\hline 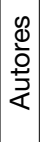 & 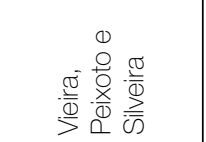 & $\begin{array}{l}\dot{\bar{\sigma}} \\
+\frac{0}{0} \\
\overline{0} \\
\dot{+}\end{array}$ & 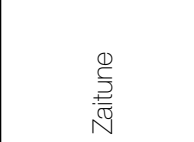 \\
\hline
\end{tabular}


Estilo de vida é considerado o modo de se viver, estando atrelado a aspectos culturais, sociais, econômicos, psicológicos e biológicos (BORGES; SEIDL, 2012; RIBEIRO et al., 2009). Pode-se afirmar que atividade física, tabagismo, manejo do estresse, consumo de álcool, hábitos alimentares, contato interpessoal, práticas religiosas, condições socioeconômicas e presença/ausência de DCNT são fatores que influenciam o estilo de vida da população idosa (BORGES; SEIDL, 2012; HARA et al., 2008; PAGOTTO; NAKATANI; SILVEIRA, 2011; PIMENTA et al., 2008; SILVA et al., 2014; ZAITUNE et al., 2007). No que diz respeito aos fatores de risco relacionados ao estilo de vida que influenciam negativamente a saúde do idoso, pode-se afirmar que inatividade física, tabagismo, presença de DCNT, alimentação inadequada, dislipidemia, consumo excessivo de álcool, obesidade, hipercolesterolemia, alterações na glicemia, resistência à insulina e o avanço da idade foram destacados (BORGES; SEIDL, 2012; COSTA et al., 2014; FERREIRA et al., 2010; HARA et al., 2008; OLIVEIRA et al., 2010; PAGOTTO; NAKATANI; SILVEIRA, 2011; PIMENTA et al., 2008; SILVA; SIMÕES; LEITE, 2007; SILVA et al., 2014; VITOI et al., 2015; VIEIRA; PEIXOTO; SILVEIRA, 2014; ZAITUNE, 2007).

A autoavaliação da saúde é um importante preditor de mortalidade (SANTIAGO, 2009). A autoavaliação negativa da saúde foi prevalente entre idosos fumantes, sedentários, com três ou mais DCNT, que fazem uso de cinco medicamentos ou mais e que não consomem frutas e verduras (PAGGOTO; NAKATANI; SILVEIRA, 2011; PIMENTA et al., 2008). No entanto, entre os idosos mais velhos, a autoavaliação positiva foi preponderante em ambos os estudos, o que foi associado à sobrevivência e às melhores condições de vida.

A presença de DCNT associa-se à avaliação negativa da saúde devido ao fato de que, quanto mais condições crônicas, mais chances do idoso referir sua saúde como razoável/ruim (JOIA; RUIZ; DONALISIO, 2007; SANTIAGO, 2009). $\mathrm{O}$ uso de medicamentos também pode indicar o estado de saúde do idoso, uma vez que, quanto mais doenças são diagnosticadas, mais medicamentos o idoso fará uso (MARIN et al., 2008; MARIN et al., 2010). 
Dentre os estudos, foram abordados diferentes fatores de risco para DCNT em idosos, tais como doenças cardiovasculares (COSTA et al., 2014; FERREIRA et al., 2010; SILVA; SIMÕES; LEITE, 2007), Diabetes Mellitus (DM) (VITOI et al., 2015) e Síndrome Metabólica (SM) (VIEIRA; PEIXOTO; SILVEIRA, 2014). De acordo com o estudo de Ferreira et al. (2010), as doenças cardiovasculares são a principal causa de morte no país, correspondendo a 38\% de óbitos entre idosos. Os fatores de risco mais prevalentes para doenças cardiovasculares encontrados neste trabalho foram: hipertensão arterial sistêmica (HAS), obesidade, DM, sedentarismo, tabagismo e consumo excessivo de álcool.

No estudo de Silva, Simões e Leite (2007), foram verificadas associações entre DM e diferentes fatores, como sedentarismo, HAS, hipercolesterolemia, obesidade e alterações na glicemia de jejum e hemoglobina glicada, que elevam o risco de mortalidade por infarto agudo do miocárdio em diabéticos. No estudo de Vitoi et al. (2015), os idosos que autorreferiram DM apresentaram diferentes fatores de risco, tais como inatividade física, sobrepeso, obesidade abdominal, HAS e dislipidemias. Ainda segundo os autores, DM é uma DCNT e também pode ser caracteriza como fator de risco cardiovascular, merecendo atenção seu controle e prevenção entre os idosos.

Segundo Costa et al. (2014), o risco de incidência de AVE dobra a cada década após os 55 anos de idade; essa doença, além do alto risco de mortalidade, traz sequelas aos sobreviventes, o que pode modificar suas atividades cotidianas. Nesse estudo, observou-se que DM foi o fator de risco mais frequente, seguida por HAS, tabagismo, dislipidemia, sedentarismo, doença cardiovascular e obesidade - fatores altamente modificáveis. Dentre os idosos, os fatores de risco não costumam ocorrer isoladamente, culminando na combinação de fatores e no aumento do risco para doenças cardiovasculares (COSTA et al., 2014; FERREIRA et al., 2010).

De acordo com Vieira, Peixoto e Silveira (2014), a SM é um conjunto de fatores para o desenvolvimento de DCNT, constituindo-se por obesidade abdominal, dislipidemia, resistência à insulina e HAS, que se acentuam com o envelhecimento. Nascimento et al. (2015) apontam o aumento de casos de HAS, DM e número de consultas médicas entre idosos brasileiros num intervalo de 10 anos, estando a HAS e a DM associadas positivamente à limitação da mobilidade. Esses dados demonstram a força de associação entre DCNT e declínio funcional.

Segundo Oliveira et al. (2010), a ocorrência de fraturas decorrentes da fragilidade óssea corresponde a importante aumento na morbimortalidade entre idosos. No estudo desses autores, optou-se por verificar a ocorrência de fratura 
vertebral por relacionar-se à osteoporose e representar risco elevado para a ocorrência de novas fraturas. Foi identificada alta prevalência, associando-se positivamente à inatividade física e ao avanço da idade.

\subsection{Atividade física}

A atividade física é caracterizada como qualquer movimento corporal em que haja gasto energético (TRIBES; VIRTUOSO JÚNIOR; OLIVEIRA, 2012), isto é, engloba desde uma Atividade de Vida Diária (AVD) (ALVES et al., 2007; DIAS et al., 2015; RIBEIRO; NERI, 2012) até um exercício físico com alto gasto energético (LIMA-SILVA et al., 2010; SANTIAGO et al., 2015). No estudo de Alves et al. (2007), a presença de doença cardíaca, artropatia, doença pulmonar e câncer encontrou-se alta entre idosos dependentes nas AVDs, que se referem às tarefas de autocuidado, como banhar-se e vestir-se, e nas Atividades Instrumentais de Vida Diária (AIVDs), que consistem nas tarefas relacionadas à vida independente em sociedade, como utilizar transporte, telefonar e preparar uma refeição. As atividades Avançadas de Vida Diária (AAVDs), que envolvem o funcionamento físico, mental e social em atividades como praticar esportes, dirigir e trabalhar, apresentou-se como fator protetor para o declínio cognitivo - quanto mais AAVDs realizadas, menor o risco de declínio. Para Freitas et al. (2010), a capacidade de realizar AAVDs tem importante papel na manutenção da capacidade respiratória. Ribeiro e Neri (2012) demonstraram que idosas sedentárias apresentam alto risco de interrupção das AAVDs e dependência para AIVDs, o que associa a prática de atividades físicas à manutenção da boa saúde e vida social desse grupo populacional.

Se tratando de inatividade física, no estudo de Alves et al. (2010) foi encontrada uma prevalência de $68 \%$ entre os idosos pernambucanos, sendo maior a partir dos 76 anos e predominante no sexo masculino, o que se assemelhou aos achados da pesquisa de Souza, Fillebaum e Blay (2015), na qual 62\% dos idosos gaúchos do sexo masculino foram classificados como inativos fisicamente. Os resultados do estudo de Zaitune et al. (2007), em que a prevalência do sedentarismo foi de $70,9 \%$ entre idosos paulistas, trouxe como diferença o fato de que a predominância ocorria no sexo feminino. Esses dados parecem ilustrar que diferenças regionais não modificam o nível de sedentarismo entre idosos no país. A pesquisa de Corrêa, Rombaldi e Silva (2011) encontrou uma prevalência de $83 \%$ de sedentarismo e alta associação com os denominados "sintomas do envelhecimento", tais como depressão, ansiedade, perda de força e massa muscular, diminuição da densidade óssea e alterações no perfil lipídico. 
Os idosos que praticam atividade física demonstram maior qualidade de vida se comparados aos idosos sedentários (FREITAS; MEEREIS; GONÇALVES, 2014), modificações positivas em variáveis antropométricas (MENEZES-CABRAL et al., 2009; SANTIAGO et al., 2015); associação inversa com demência e depressão (BENEDETTI et al., 2008); melhor desempenho em funções cognitivas (LIMA-SILVA et al., 2010; VARGAS; LARA; MELLO-CARPES, 2014) e mesmo desempenho em habilidade motora que jovens (GEHRING et al., 2009). No entanto, mesmo sendo as informações relacionadas aos benefícios dessa prática constantes e veementes (BENEDETTI et al., 2008; CORREAA; ROMBALDI; SILVA, 2011), no presente estudo, foi possível verificar alto grau de sedentarismo entre os idosos brasileiros (ALVES et al., 2010; CORREAA; ROMBALDI; SILVA, 2011; SOUZA; FILLEBAUM; BLAY, 2015; ZAITUNE et al., 2007).

De modo geral, se manter ativo fisicamente demonstra-se necessário para uma boa saúde entre idosos. Segundo Alves et al. (2007), preservar independência na realização de AVDs e AIVDs pode indicar menor acometimento por doenças cardiorrespiratórias e articulares. A atividade física é reconhecida como objetivo de saúde pública (FREITAS; MEEREIS; GONÇALVES, 2014), uma vez que é considerada fator de proteção sobre diversas DCNT, tais como as cardiovasculares, osteoporose, cânceres, diabetes e depressão (BENEDETTI et al., 2008; COELHO et al., 2014; CORRÊA; ROMBALDI; SILVA, 2011; MENEZES-CABRAL et al., 2009; SOUZA; FILLENBAUM; BLAY, 2015). Além disso, auxilia na prevenção e tratamento de déficits de memória (VARGAS; LARA; MELLO-CARPES, 2014) e demência (BENEDETTI et al., 2008), diminui o risco de morte precoce em indivíduos com elevado gasto energético e bom condicionamento físico (CORRÊE; ROMBALDI; SILVA, 2011) e é importante frente à Síndrome da Fragilidade (TRIBES; VIRTUOSO JÚNIOR; OLIVEIRA, 2012).

\subsection{Alimentação}

Alimentação e nutrição apresentam aspectos diferentes: nutrição constrói-se em torno do quantificável, envolvendo a energia e os nutrientes necessários para o corpo humano; alimentação consiste em um ato biológico envolto de cultura e subjetividade, que, ao mesmo tempo em que atende à fome, relaciona-se com crenças e sensações, indicando o que é considerado comestível ou não (MENEZES et al., 2015). Assim, é possível afirmar que a alimentação somente exerce papel positivo na saúde se apresentar aspectos nutricionais apropriados (ALENCAR; BARROS JÚNIOR; CARVALHO, 2008; CAVALCANTI et al., 2011).

Segundo Fares et al. (2012), o estado nutricional do idoso depende de diversos fatores, tais como renda (acesso a alimentos), hábito de fumar, período 
na posição sentada, e presença de patologias, como HAS. Menezes et al. (2015) verificaram em seu estudo que a alimentação saudável impacta de forma positiva na prevenção de DCNT, como doenças cardiovasculares, diabetes e câncer. Alencar, Barros Júnior e Carvalho (2008) apontam a necessidade da educação nutricional para o envelhecimento saudável, uma vez que hábitos alimentares repercutem sobre o metabolismo do organismo envelhecido e, segundo Cavalcanti et al. (2011), a nutrição adequada é um dos fatores que determinam o potencial de longevidade (CAVALCANTI et al., 2011).

É fundamental ressaltar também que o tabagismo entre idosos é um importante problema de saúde. De acordo com Costa et al. (2014), o hábito de fumar aumenta de duas a quatro vezes as chances de ser acometido por um AVE. A interrupção do tabagismo em qualquer idade traz diversos benefícios, como redução do risco de doença e morte, aumento na expectativa de vida, maior controle de doenças preexistentes e melhoria na condição geral de saúde (CARVALHO; GOMES; LOUREIRO, 2010).

\section{Considerações finais}

A partir desse estudo é possível afirmar que os idosos brasileiros apresentam estilo de vida inadequado e diferentes fatores de risco, culminando em baixas condições de saúde e autoavaliações negativas da saúde, mesmo esses fatores de risco sendo, em sua maioria, altamente modificáveis. Diante disto, percebe-se a urgente necessidade de se atuar de maneira preventiva frente às consequências de um estilo de vida inadequado. Educação em saúde, acesso a alimentos saudáveis e a manutenção de uma vida sempre ativa são meios plausíveis de prevenção, como foi verificado ao longo do trabalho. Cabe, pois, analisar a realidade de cada população a se intervir, mas o panorama nacional apresentado nesta pesquisa denota semelhança entre o estilo de vida e os fatores influentes na saúde dos idosos nas diferentes regiões do país.

Vale ressaltar, como restrição do presente estudo, a delimitação dos idiomas português e inglês e a utilização de apenas um portal e de uma base de dados. Apesar de o Portal BVS ter amplo alcance de publicações, alguns estudos considerados importantes podem ter sido excluídos. 


\title{
abstract
}

Population aging is a fact and many are the factors that influence the health of individuals who are aging, such as lifestyle, which is one of the most important items to present good health. Considering the increase of the old population in Brazil and the great influence of lifestyle on the health of the elderly, it was conducted an integrative review to identify which factors influence the lifestyle of the Brazilian elderly and what the consequences for their health according to the national scientific production. The search was based on Virtual Health Library (VHL) and the Scientific Electronic Library Online (SciELO), from the combination of the descriptors "aging", "aged" and "lifestyle". Research published between 2006 and 2016 were selected, in English and Portuguese, were included. Thirty-nine studies were consistent with the inclusion criteria. It was found that physical inactivity, smoking, presence of Chronic Non-Communicable Disease, poor nutrition, dyslipidemia, binge drinking, obesity, hypercholesterolemia, glycemia alterations, insulin resistance and age advance were the main risk factors related to lifestyle that affect the elderly health. The negative health self-assessment was common in elderly smokers and sedentary, affected by three or more Chronic Non-Communicable Disease that use five or more medicines and not consumer fruits and vegetables. The conclusion is that the Brazilian elderly population presents inadequate lifestyle and several risk factors, resulting in reduced health conditions and negative self-assessments of health.

keywords

Health of the Elderly. Life Style. Risk Factors.

referências

\begin{abstract}
ALENCAR, Maria do Socorro; BARROS JÚNIOR, Francisco; CARVALHO, Cecília. Os aportes sócio-políticos da educação nutricional na perspectiva de um envelhecimento saudável. Revista de Nutrição, Campinas, v. 21, n. 4, p. 369-381, jul./ago. 2008.

ALVES, João Guilherme et al. Prevalência de adultos e idosos insuficientemente ativos moradores em áreas de unidades básicas de saúde com e sem Programa Saúde da Família em Pernambuco, Brasil. Cadernos de Saúde Pública, Rio de Janeiro, v. 26, n. 3, p. 543-556, mar. 2010.
\end{abstract}


ALVES, Luciana Correia et al. A influência das doenças crônicas na capacidade funcional dos idosos do Município de São Paulo, Brasil. Cadernos de Saúde Pública, Rio de Janeiro, v. 23, n. 8, p. 1924-1930, ago. 2007.

BENEDETTI, Tânia et al. Atividade física e estado de saúde mental de idosos. Revista de Saúde Pública, São Paulo, v. 42, n. 2, p. 302-307, abr. 2008.

BORGES, Lilian Maria; SEIDL, Elaine Maria. Percepções e Comportamentos de Cuidados com a Saúde Entre Homens Idosos. Psicologia: Ciência e Profissão, Brasília, v. 32, n. 1, p. 66-81, out. 2012

CARVALHO, Anderson; GOMES, Lucy; LOUREIRO, Altair. Tabagismo em idosos internados em instituições de longa permanência. Jornal Brasileiro de Pneumologia, Brasília, DF, v. 36, n. 3, p. 339-346, mar. 2010.

CAVALCANTI, Christiane et al. Programa de intervenção nutricional associado à atividade física: discurso de idosas obesas. Ciência e Saúde Coletiva, Rio de Janeiro, v. 16, n. 5, p. 2383-2390, maio 2011.

COELHO, Bruna et al. Comparação da força e capacidade funcional entre idosos praticantes de musculação, hidroginástica e não praticantes de exercícios físicos. Revista Brasileira de Geriatria e Gerontologia, Rio de Janeiro, v. 17, n. 3, p. 497-504, jul./set. 2014.

CORRÊA, Leandro; ROMBALDI, Airton; SILVA, Marcelo. Atividade física e sintomas do envelhecimento masculino em uma população do Sul do Brasil. Revista Brasileira de Medicina do Esporte, São Paulo, v. 17, n. 4, p. 228-231, jul./ago. 2011.

COSTA, Viviane et al. Prevalence of risk factors for the occurrence of strokes in the elderly. Fisioterapia em Movimento, Curitiba, v. 27, n. 4, p. 555-563, out./dez. 2014

DIAS, Eliane et al. Atividades avançadas de vida diária e incidência de declínio cognitivo em idosos: Estudo SABE. Cadernos de Saúde Pública, Rio de Janeiro, v. 31, n. 8, p. 1623-1635, ago. 2015.

FARES, Daniele et al. Fatores associados ao estado nutricional de idosos de duas regiões do Brasil. Revista da Associação Médica Brasileira, São Paulo, v. 58, n. 4, p. 434-441, jul./ago. 2012.

FAZZIO, Débora Mesquita. Envelhecimento e qualidade de vida - uma abordagem nutricional e alimentar. Revista de Divulgação Científica Sena Aires, Valparaíso de Goiás, v. 1, n. 1, p. 76-88, jun. 2012.

FERREIRA, Carla Cristina et al. Prevalência de fatores de risco cardiovascular em idosos usuários do Sistema Único de Saúde de Goiânia. Arquivos Brasileiros de Cardiologia, São Paulo v. 95, n. 5, p. 621-628, nov. 2010.

FOCCHESATTO, Andréia; ROCKETT, Fernanda; PERRY, Ingrid. Risk and protective factors for the development of chronic diseases in a rural elderly population in Rio Grande do Sul. Revista Brasileira de Geriatria e Gerontologia, Rio de Janeiro, v. 18, n. 4, p. 779-795, out./dez. 2015.

FREITAS, Caroline; MEEREIS, Estele; GONÇALVES, Marisa. Qualidade de vida de idosos ativos e insuficientemente ativos do município de Santa Maria (RS). Revista Kairós Gerontologia, São Paulo, v. 17, n. 1, p. 57-68, mar. 2014.

FREITAS, Fábia et al. Relação entre força de tosse e nível funcional em um grupo de idosos. Revista Brasileira de Fisioterapia, São Carlos, v. 14, n. 6, p. 470-476, nov./dez. 2010.

GEHRING, Paula et al. Desempenho de idosos em uma tarefa motora de demanda dupla de controle. Revista Brasileira de Educação Física e Esporte, São Paulo, v. 23, n. 3, p. 211-220, jul./set. 2009.

GONZALEZ, Lilian Maria; SEIDL, Eliane Maria. O envelhecimento na perspectiva de homens idosos. Paidéia, Ribeirão Preto, v. 21, n. 50, set./dez. 2011. 
HARA, Cláudia et al. Prevalence of excessive daytime sleepiness in Brazilian community-dwelling older adults with very low levels of schooling, and its association with sociodemographic characteristics and lifestyle: the Bambuí Health and Ageing Study (BHAS). Jornal Brasileiro de Psiquiatria, Rio de Janeiro, v. 57, n. 2, p. 91-97, jun. 2008.

INSTITUTO BRASILEIRO DE GEOGRAFIA E ESTATÍSTICA (IBGE). Pesquisa nacional de saúde 2013: percepção do estado de saúde, estilos de vida e doenças crônicas. Rio de Janeiro: IBGE, 2014

JOBIM, Fátima Angelina; JOBIM, Eduardo. Atividade Física, Nutrição e Estilo de Vida no Envelheicmento. UNOPAR Científica: Ciências Biológicas e da Saúde, Londrina, V. 17, n. 4, p. 298-308, 2015

JOIA, Luciane; RUIZ, Tânia; DONALISIO, Maria Rita. Condições associadas ao grau de satisfação com a vida entre a população de idosos. Revista de Saúde Pública, São Paulo, v. 41, n. 1, p. 131-138, fev. 2007

LIMA-SILVA, Thaís et al. Fluência verbal e atividade física no processo de envelhecimento normal: um estudo epidemiológico em Ermelino Matarazzo, São Paulo, Brasil. Revista Brasileira de Atividade Física e Saúde, São Paulo, v. 15, n. 1, p. 50-56, fev. 2010.

MARIN, Maria José et al. Caracterização do uso de medicamentos entre idosos de uma unidade do Programa Saúde da Família. Cadernos de Saúde Pública, Rio de Janeiro, v. 24, n. 7. p. 1545-1555, jul. 2008.

MARIN, Maria José et al. Diagnóstico de enfermagem de idosos que utilizam múltiplos medicamentos. Revista da Escola de Enfermagem da USP, São Paulo, v. 44, n. 1, p. 47-52, mar. 2010

MENEZES, Maria Fátima et al. Reflexões sobre alimentação saudável para idosos na agenda pública brasileira. Revista Brasileira de Geriatria e Gerontologia, Rio de Janeiro, v. 18, n. 3, p. 599-610, jul./set. 2015.

MENEZES-CABRAL, Roberto Luiz et al. Efeitos de diferentes treinamentos e estilos de vida nos indicadores antropométricos e cardiocirculatórios no envelhecimento. Revista de Salud Pública, Bogotá, v. 11, n. 3, p. 359-369, abr. 2009.

NASCIMENTO, Clarissa et al. Diabetes, hypertension and mobility among Brazilian older adults: findings from the Brazilian National Household Sample Survey $(1998,2003$ and 2008). BioMed Central Public Health, v. 15, n. 591, p. 1-7, June 2015.

OLIVEIRA, Patrícia et al. Prevalência de fraturas vertebrais e fatores de risco em mulheres com mais de 60 anos de idade na cidade de Chapecó, Santa Catarina, Brasil. Cadernos de Saúde Pública, Rio de Janeiro, v. 26, n. 9, p. 1777-1787, set. 2010.

ORGANIZAÇÃO MUNDIAL DE SAÚDE (OMS). O papel da atividade física no Envelhecimento saudável. Florianópolis: OMS, 2006

PAGOTTO, Valeria; NAKATANI Adelia; SILVEIRA, Érika. Fatores associados à autoavaliação de saúde ruim em idosos usuários do Sistema Único de Saúde. Cadernos de Saúde Pública, Rio de Janeiro, v. 27, n. 8, p. 1593-1602, ago. 2011.

PETROSKI, E. L.; PELEGRINI, A. Associação entre o estilo de vida dos pais e a composição corporal dos filhos adolescentes. Revista Paulista de Pediatria, São Paulo, v. 27, n. 1, p. $48-52,2009$

PIMENTA, Fausto et al. Avaliação da qualidade de vida de aposentados com a utilização do questionário SF-36. Revista da Associação Médica Brasileira, São Paulo, v. 54, n. 1, p. 55-60, jan./fev. 2008

RIBEIRO, Luciana; NERI, Anita. Exercícios físicos, força muscular e atividades de vida diária em mulheres idosas. Ciência e Saúde Coletiva, Rio de Janeiro, v. 17, n. 8, p. 2169-2180, ago. 2012 
RIBEIRO, Pricila et al. Variabilidade no envelhecimento ativo segundo gênero, idade e saúde. Psicologia em Estudo, Maringá, v. 14, n. 3, p. 501-509, jul./set. 2009.

SANTIAGO, Lívia Maria. Condições associadas à saúde auto-referida [sic] em homens idosos de Juiz de Fora, MG. 2009. Dissertação (Mestrado em Saúde Pública e Meio Ambiente) - Fundação Oswaldo Cruz - Escola Nacional de Saúde Pública Sergio Arouca, Rio de Janeiro, 2009.

SANTIAGO, Luís Ângelo et al. Treinamento resistido reduz riscos cardiovasculares em idosas. Revista Brasileira de Medicina do Esporte, São Paulo, v. 21, n. 4, p. 261-265, jul./ago. 2015.

SILVA, Henrique et al. Correlates of above-average cognitive performance among older adults: the SABE study. Cadernos de Saúde Pública, Rio de Janeiro, v. 30, n. 9, p. 1977-1986, set. 2014.

SILVA, Rodrigo; SIMÕES, Maria Jacira; LEITE, Amauri. Fatores de risco para doenças cardiovasculares em idosos com diabetes mellitus tipo 2. Revista de Ciências Farmacêuticas Básica e Aplicada, v. 28, n. 1, p. 113-121, 2007.

SOUZA, Adelle; FILLENBAUM, Gerda; BLAY, Sérgio. Prevalence and correlates of physical inactivity among older adults in Rio Grande do Sul, Brazil. Plos One, Durhan, v. 10, n. 2, p. 1-14, fev. 2015.

SOUZA, Marcela; SILVA, Michelly; CARVALHO, Rachel. Revisão integrativa: o que é e como fazer. Einstein, São Paulo, v. 8, n. 1, p. 102-106, mar. 2010.

SOUZA, Marilza Amaral Henrique de et al. Perfil do estilo de vida de longevos. Revista Brasileira de Geriatria e Gerontologia, Rio de Janeiro, v. 19, n. 5, p. 819-826, 2016.

TRIBES, Sheilla; VIRTUOSO JÚNIOR, Jair; OLIVEIRA, Ricardo. Atividade física como preditor da ausência de fragilidade em idosos. Revista da Associação Médica Brasileira, São Paulo, v. 58, n. 3, p. 341-347, maio/jun. 2012.

VARGAS, Liane; LARA, Marcus; MELLO-CARPES, Pâmela. Influência da diabetes e a prática de exercício físico e atividades cognitivas e recreativas sobre a função cognitiva e emotividade em grupos de terceira idade. Revista Brasileira de Geriatria e Gerontologia, Rio de Janeiro, v. 17, n. 4, p. 867-878, jul. 2014.

VIEIRA, Edna; PEIXOTO, Maria do Rosário; SILVEIRA, Erika. Prevalência e fatores associados à Síndrome Metabólica em idosos usuários do Sistema Único de Saúde. Revista Brasileira e Epidemiologia, São Paulo, v. 17, n. 4, p. 805-817, out./dez. 2014.

VITOI, Nayla et al. Prevalência e fatores associados ao diabetes em idosos no município de Viçosa, Minas Gerais. Revista Brasileira e Epidemiologia, São Paulo, v. 18, n. 4 p. 953-965, out./dez. 2015

WORLD HEALT ORGANIATION (WHO). The world health report 2013: research for universal health coverage. Luxembourg: WHO, 2013.

ZAITUNE, Maria Paula et al. Fatores associados ao sedentarismo no lazer em idosos. Campinas, São Paulo, Brasil. Cadernos de Saúde Pública, Rio de Janeiro, v. 23, n. 6, p. 1329-1338, jun. 2007.

Data de Submissão: 03/07/2017

Data de Aprovação: 25/04/2018 
